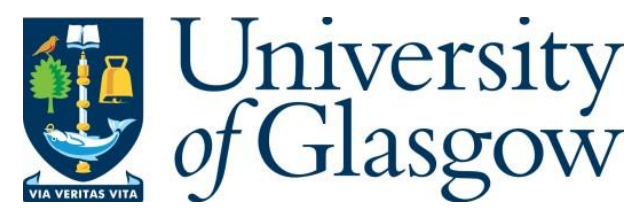

MacKenzie, R., Monaghan, L., Masson, R. A., Werner, A. K., Caprez, T. S., Johnston, L. and Kemi, O. J. (2019) Physical and physiologic determinants of rock climbing. International Journal of Sports Physiology and Performance, (doi:10.1123/ijspp.20180901).

There may be differences between this version and the published version. You are advised to consult the publisher's version if you wish to cite from it.

http://eprints.gla.ac.uk/185251/

Deposited on: 25 April 2019

Enlighten - Research publications by members of the University of Glasgow http://eprints.gla.ac.uk 


\title{
Physical and Physiologic Determinants of Rock Climbing
}

Original Investigation

Robert MacKenzie, Linda Monaghan, Robert A Masson, Alice K Werner, Tansinee S Caprez, Lynsey Johnston, Ole J Kemi

School of Life Sciences and Institute of Cardiovascular and Medical Sciences; College of Medical, Veterinary and Life Sciences; University of Glasgow, Glasgow, UK.

Corresponding author: Dr Ole J Kemi, School of Life Sciences, Sir James Black Building, University of Glasgow, Glasgow, G12 8QQ, UK

E-mail: ole.kemi@glasgow.ac.uk; Phone: +44 (0)141 3305962

\section{Running head: Determinants of Climbing Performance}

\author{
Abstract word count: 250 \\ Text-only word count: 3446 \\ Number of figures and tables: 6 figures and 3 tables \\ Number of references: 30
}


Purpose-Rock climbing performance relies on many characteristics. Here, we identified the physical and physiologic determinants of peak performance in rock climbing across the range from lower-grade to elite.

Methods-44 male and 33 female climbers with onsight maximal climbing grades 5a-8a and 5a$7 \mathrm{~b}+$, respectively, were tested for physical, physiologic and psychologic characteristics (independent variables) that were correlated and modelled by multiple regression and principal component analysis to identify the determinants of rock climbing ability.

Results-In males, 23 of 47 variables correlated with climbing ability ( $p<0.05$, Pearson's correlation coefficients 0.773-0.340), including shoulder endurance, hand and finger strength, shoulder power-endurance, hip flexibility, lower-arm grip strength, shoulder power, upper-arm strength, core-body endurance, upper-body aerobic endurance, hamstrings and lower-back flexibility, aerobic endurance, and open-hand finger strength. In females, 10 of 47 variables correlated with climbing ability ( $p<0.05$, Pearson's correlation coefficients $0.742-0.482)$ : shoulder endurance and power, lower-arm grip strength, balance, aerobic endurance, and arm span. Principal component analysis and univariate multiple regression identified the main explanatory variables. In both sexes, shoulder power and endurance measured as maximum pull-ups, average arm crank power, and bent-arm hang, emerged as the main determinants ( $p<0.01$; adjusted $R^{2}=0.77$ in males and 0.62 in females). In males, finger pincer ( $p=0.07$ ) and grip strength also had trends $(p=0.09)$ toward significant effects. Finally, in test-of-principle training studies, we trained to increase main determinants $42-67 \%$; this improved climbing ability 2-3 grades.

Conclusions-Shoulder power and endurance majorly determines maximal climbing. Finger, hand and arm strength, core-body endurance, aerobic endurance, flexibility and balance are important secondary determinants.

Keywords Strength, Endurance, Power, Flexibility, Anthropometry

.

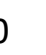


Climbing has gained popularity standards, and Olympic recognition, but not the scientific attention that other sports have. As a sport, climbing takes many forms. For recreational, competitive, and exercise training purposes, sport climbing has evolved as the widest reaching subdiscipline. ${ }^{1}$ The safe and controlled environment of fixed protection (bolts or topropes) of sport climbing allows a full focus on athletic and gymnastic challenges, ${ }^{2,3}$ and as such, it is characterized by sustained, repetitive, and complex bouts of intense upward motion that tax physical capacity in the upper-limbs and upper- and core-body. ${ }^{4,5}$ Growth of indoor facilities has further facilitated this trend. ${ }^{1}$

Thus, the current study is based upon the notion that the highest level of sport climbing ability and performance is at least partly determined by the limits of those characteristics. Accordingly, changes to those affect maximal climbing performance. Previous studies have identified many of those characteristics, such as upper-body and shoulder strength ${ }^{6-8}$ including explosive power, ${ }^{5,6,9}$ forearm grip and finger strength, ${ }^{5,10-13}$ upper-body endurance capacity ${ }^{10,14}$ and local muscle aerobic oxidative and post-occlusion re-oxygenation capacity ${ }^{12,15,16}$ as well as anthropometric characteristics and body composition and mass. ${ }^{17-18}$ Other studies have however suggested forearm grip and finger strength,, 7,17,19 anthropometric factors and body composition, ${ }^{5,20}$ flexibility ${ }^{20}$ and aerobic capacity and maximal oxygen uptake $\left(\mathrm{VO}_{2 \max }\right) .{ }^{21}$ may be less important. However, most of the above studies have assessed only a limited number of determinants; addressed cohorts with limited ability ranges, not always used sports-specific methodology, mostly excluded or under-represented females, and rarely established the relative importance of individual characteristics compared to others. For instance, the two sexes may not share some or any characteristics. ${ }^{11}$ Therefore, information on physical and physiologic parameters that dictate progress in climbing remains incomplete, such that designing informed and evidence-based specific training programs for specific purposes and cohorts becomes difficult, to the degree that this unmet need hinders optimal progress in both recreational and professional athletes trying to reach their potential.

The aim of this study was, therefore, in a comprehensive testing regime in both sexes and across the complete and widest available spectrum of climbers from lower-grade to elite standard and in a standardized and unbiased manner, to assess the physical and physiologic factors that may dictate climbing performance, to thereby identify the relative importance of each factor for determining climbing ability and performance in sport climbing.

5

6

107

(1)

9

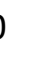


Subjects

44 males and 33 females volunteered as subjects (table 1). They were screened for maximal onsight (complete a climb on first attempt) rock climbing ability in order to balance number of subjects in each sport climbing grade; the French sport climbing grade scale was used and transformed to a linear scale to allow for statistical modelling $(5 a=1,5 a+=2 \ldots)$. This resulted in males: 3-4 subjects/grades 5a-7c and 1 for grades $7 c+$ and $8 a$; females: 3-4/grades 5a-7a, 2 for grades $7 a+$ and $7 b$ and 1 for grade $7 b+$.

The Institutional Review Board approved the study and it was performed in accordance with the Declaration of Helsinki. Subjects were health screened and signed informed consent forms prior to commencement. Exclusion criteria included regular smoking, medication, and pre-existing medical conditions contraindicative to exercise testing. Subjects were asked to avoid exhaustive exercise and alcohol within 48 hours and food and fluids except water within 2 hours of each test, each separated by a week, and all subjects were familiarized with the equipment and protocols. The dominant limb was used for single-limb tests. ${ }^{11,20}$

\section{Design}

Observational cross-sectional and prospective research.

\section{Methodology}

Climbing ability was self-reported as the highest consistently completed indoor onsight grade and confirmed or assessed if unknown by a 12-15m onsight test-climb during top-rope conditions (Glasgow Climbing Centre, Glasgow, UK), secured and belayed by certified climbing instructors. Self-reported and assessed grades were in agreement, as previously reported. ${ }^{22}$ Immediately before the climb, subjects completed a Competitive State Anxiety Inventory-2 (CSAI-2) questionnaire, ${ }^{23-}$ 24 to assess self-confidence and cognitive and somatic anxiety.

Body height and weight, leg and arm lengths, arm span, and finger and hand lengths, and resting blood pressure were measured, with body mass index (BMI) and "ape index" (arm span/body height) calculated. ${ }^{5,12,20}$

Hip flexibility was measured by the foot-raise test, with the subject standing next to a wall with straight arms extended to $90^{\circ}$ and palms flat on the wall and then lifting the right foot as high as possible, ${ }^{11}$ and by a leg-span test by measuring the maximum achievable distance between feet. ${ }^{11}$ Hamstring/lower-limb and lower-back flexibility was measured by a sit-and-reach box (HaBdirect, Southam, UK) test where the subject from a sitting position and straight legs reached forward as far as possible to hold for 3 seconds. ${ }^{11}$

Balance was measured by the subject standing on the balls (metatarsal) of one foot for as long as possible until failure.

Hand-eye spatial coordination was measured by the subject holding a card in front of them and marking a dot on the front. They then made 3 attempts to mark a dot on the back of the card as close to the original dot as possible, with the average distance between front and back dots recorded. Foot-eye spatial coordination was measured by the subject standing on one foot on a climbing wall, with the other foot hanging, a forward-pointing marker attached underneath, and a card placed $30 \mathrm{~cm}$ diagonally up. The subject marked a dot on the card and made 3 attempts to mark another dot as close to the original as possible, with the average distance from the target recorded.

Arm bicep (upper-arm) and grip (lower-arm), hand and finger pincer and open hand finger strength were measured during isometric maximal voluntary contractions (3 5-second efforts), 
whereby the highest maximum force was recorded (PrimusRS, BTE Technologies, Hanover, CO). During each test, the subject stood with the elbow at $90^{\circ}$, and was instructed to grip (arm grip test) and supine grip (bicep test) a gauge, pinch a plate between thumb and other digits (pincer test), and push down a plate with extended fingers (open-hand finger test). The dynamometer has been validated for similar purposes. ${ }^{25}$

Shoulder power and power-endurance was recorded, by lying in a supine position while turning the PrimusRS arm crank ergometer against isotonic resistance at $20 \%$ of maximum isometric force, for a 20 -second maximum effort. The maximum isometric force, maximum and average power, and power decline as a measure of power-endurance were recorded.

Measurements of climbing-specific upper-body, shoulder, and core-body endurance and power were made using a pull-up board (Beastmaker 1000, Beastmaker, Sheffield, UK). ${ }^{11,17}$ A leg-raise hang-test recorded maximum hanging time with stretched legs lifted to $90^{\circ}$ of the body; a bentarm hang-test recorded the maximum time hanging from the pull-up board with elbows at $90^{\circ}$, and a maximum pull-up test recorded the maximum number of pull-ups from full arm extension to chin above board.

For aerobic capacity, an incremental exercise test measured $\mathrm{VO}_{2 \max }$ by an exhaustive ramp treadmill (PPS Med, Woodway, Weil am Rhein, Germany) test. ${ }^{26}$ With the speed fixed at $8 \mathrm{Km} / \mathrm{h}$, the gradient increased by $2 \%$ every 2 minutes until volitional exhaustion. Similarly, peak oxygen uptake $\left(\mathrm{VO}_{2 \text { peak }}\right)$ was measured during upper-body exercise using an arm crank ergometer (Top XT, Technogym, Bracknell, UK), with speed at 60rpm and resistance increasing every minute.

\section{Training Study}

Finally, we recruited 6 male (27.3 \pm 3.1 years, $173.2 \pm 6.5 \mathrm{~cm}, 68.0 \pm 3.2 \mathrm{~kg}$; climbing grades $6 \mathrm{a}-6 \mathrm{c}$ ) and 6 female $(26.5 \pm 2.6$ years, $162.1 \pm 3.7 \mathrm{~cm}, 63.1 \pm 3.9 \mathrm{~kg}$; climbing grades $5 \mathrm{a}-6 \mathrm{~b}+)$ climbers: males trained maximum pull-ups $(n=3)$ and balance $(n=3)$, while females trained bent-arm hangs $(n=3)$ and leg-raise hangs ( $n=3$; the respective main determinants and variables that only insignificantly showed weak trends to correlate with climbing ability). Training was performed as 3 sets to failure with 4-minute breaks, 2/week for 4 weeks, with climbing training continuing at will. Pre-tests and post-tests including climbing ability were measured as described above.

\section{Statistics}

Climbing ability was set as the dependant variable, whereas other variables were treated as independent. Pearson's correlation coefficients assessed the relationships between the dependent and independent variables. Next, a principal component analysis (PCA), with components extracted by eigenvalue $>1$ (Kaiser criterion and Varimax rotation) and multiple univariate stepwise linear regression was constructed to model and attribute relative importance to each individual independent variable for determining the variability in the dependent variable (climbing ability); both forward and backward stepwise modes for elimination of insignificant variables were applied. Training effects were evaluated by the Wilcoxon signed rank test. Throughout, significance was set at $p<0.05$. Data were normally distributed (Shapiro-Wilks test of normality) and are expressed as means \pm standard deviation (SD). Statistical analysis was computed by SPSS
version 24 (IBM, Armonk, NY). and are expressed as means \pm standard deviation (SD). Statistical analysis was computed by SPSS
version 24 (IBM, Armonk, NY). 


\section{Correlations Between Climbing Ability and Independent Variables}

207 In males (climbing ability 5a-8a), 23 of the 47 ( 50\%) independent variables statistically 208 significantly correlated with climbing ability (Table 2), with Pearson's correlation coefficients $0.773-0.340$, while 5 variables showed trends toward statistical significance $(p=0.05-0.1)$.

In females (climbing ability 5a-7b+), 10 of the 47 ( 20\%) independent variables statistically significantly correlated with climbing ability (Table 3), with Pearson's correlation coefficients $0.742-0.482$, while 3 variables showed trends toward statistical significance ( $p=0.05-0.1$ ). Thus, $\sim 30 \%$-age points ( 50\%- 20\%) fewer parameters of physical and physiologic capacity correlated with climbing ability in females versus males.

\section{Upper-Body, Shoulder and Core-Body Endurance and Power}

Climbing ability showed the closest relationship to climbing-specific upper-body and shoulder endurance and power, measured by the maximum pull-ups and bent-arm hang-time, including after body weight normalization, as assessed by linear regression (Figure 1A-D) and linear correlation (Tables 2,3).

In males, a close relationship between climbing ability and maximum pull-ups and bent-arm hangtime was followed by a close relationship between climbing ability and measurements of shoulder power, albeit with lower linear regression goodness-of-fit and Pearson's correlation coefficients for both average and maximum power during a 20-second arm crank test (Figure 1E,G, Table 2). In females, in contrast, 20-second average arm crank power normalized for body weight showed only a trend toward significant correlation to climbing ability (Table 3), whereas linear regression showed a trend toward statistical significance between climbing ability and average 20 -second power (Figure 1F; normalized for body weight (Figure 1F insert): linear regression goodness-of-fit $\mathrm{r}^{2}=0.106, p=0.06$ ). Other measurements of shoulder power measured during the arm crank test did not show a significant relationship to climbing ability in females (Figure 1H, Table 3).

The ability to sustain power (arm crank 20-second power decline) did not show a significant relationship with climbing ability in either sex (Tables 2,3; linear regression not shown).

Core-body endurance measured as maximum leg-raise hang-time was also found to correlate significantly with climbing ability in males (Figure 1I, Table 2), but not females (Figure 1J, Table 3 ), though a trend toward statistical significance occurred, including after normalization to body weight (Figure 1J insert: linear regression goodness-of-fit $\mathrm{r}^{2}=0.095, p=0.08$ ).

\section{Arm, Hand, and Finger Strength}

In males, climbing ability showed a significant relationship with all measurements of arm, hand, and finger strength (Table 2): upper-arm bicep strength (Figure 2A), lower-arm grip strength (Figure 2C), hand and finger pincer strength (Figure 2E), and open-hand finger strength (Figure 2G), including after normalization to body weight (Figure 2A,C,E,G inserts).

In contrast, females showed a weaker relationship between climbing ability and arm, hand, and finger strength (Table 3), and only lower-arm grip strength showed a significant relationship with climbing ability (Figure 2D), including after normalization to body weight (Figure 2D insert), However, upper-arm bicep strength showed a trend towards statistical significance to climbing ability (Figure 2B), including after normalization to body weight (Figure 2B insert: $p=0.08$ ). 
Aerobic endurance capacity in both upper- and whole-body ( $\mathrm{VO}_{2 \text { peak }}$ and $\mathrm{VO}_{2 \text { max }}$ respectively), showed a statistically significant relationship with climbing ability in both sexes (Tables 2,3, Figure 3).

\section{Flexibility, Balance, and Coordination}

In males, hip, hamstring/lower-limb and lower-back flexibility showed a statistically significant relationship with climbing ability (Table 2, Figure 4A,C), whereas the foot-raise test; also a measure of hip flexibility, did not correlate significantly with climbing ability (Table 2). In contrast, no measurements of flexibility showed a significant relationship with climbing ability in females (Table 3, Figure 4B,D).

Balance correlated significantly with climbing ability in females (Table 3), including a significant linear regression goodness-of-fit (Figure 4F), whereas in males, only a trend toward statistical significance occurred (Table 2, Figure 4E).

Neither of hand-eye or foot-eye spatial coordination showed a significant relationship to climbing ability in either sex (Tables 2,3).

\section{Body Dimensions}

Body weight, BMI, or body height did not correlate significantly with climbing ability in either sex (Tables 2,3). However, body height showed a weak trend towards a significant linear regression in males (Figure 5C) and females (Figure 5D), respectively.

Arm span showed a weak, but insignificant trend towards correlation (Table 2), but a significant linear regression (Figure 5E) with climbing ability in males. In females, "ape index," but not arm span showed a significant linear correlation (Table 3) and regression (Figure 5F) with climbing ability; however, it should be noted, the slope was negative and with a very small coefficient of increment (-0.002843 \pm 0.0009281$)$.

Measurements of arm, hand, finger and leg lengths did not show significant relationships with climbing ability in either sex (Tables 2,3).

\section{Anxiety and Self-Confidence During Climbing}

Measurements of cognitive or somatic anxiety or self-confidence showed detrimental levels, but did not discriminate between different levels of ability in either sex (Tables 2,3), though a weak trend toward a significant correlation between self-confidence and climbing ability occurred in males (Table 2).

\section{Principal Component Analysis and Multiple Univariate Linear Regression}

PCA and forward multiple regression identified the main explanatory variables that determined the variation in climbing ability. In males, shoulder power and endurance, i.e. maximum pull-ups, average arm crank power, and bent-arm hang emerged as the main determinants, explaining in total $77 \%$ (59\%, 14\%, and 4\% individually) of the variation (Figure 6A). Pincer and grip strength had trends $(p=0.07$ and $p=0.09)$ toward significant effects, while in a model only including nontrainable variables to assess the relative importance of body dimensions, hand length emerged as the only significant explanatory variable (unstandardized coefficient $b 0.70 \pm$ standard error (SE) 0.78 , adjusted $R^{2} 0.08(p=0.04)$, residual SD 3.52), whereas arm span had a trend $(p=0.09)$ toward a significant effect.

In females, shoulder power and endurance, i.e. bent-arm hang and maximum pull-ups also emerged as the main determinants for climbing ability, explaining in total $62 \%$ (54\%, and 8\% 
individually) of the variation (Figure 6B), while the "ape index" emerged as the only significant explanatory variable when modelling non-trainable body dimension variables (unstandardized coefficient $b-78.44 \pm \mathrm{SE} 25.88$, adjusted $R^{2} 0.21$ ( $\left.p=0.01\right)$, residual SD 2.59).

Backward regression, multiple regression with or without variables (principle components) identified by eigenvalue $>1$ by PCA and a model only including trainable variables yielded similar results (not shown).

\section{Training Study}

Finally, we trained the main determinants, maximum pull-ups in males and bent-arm hangs in females, to increase $42 \%$ and $67 \%$, respectively. Upon this, climbing ability increased $2 \pm 1$ and $2.7 \pm 1.5$ grades, respectively (Figures $6 \mathrm{C}-\mathrm{D}$ ). We also trained variables without significant correlation to climbing ability; balance in males and leg-raise hang in females, both with comparable insignificant correlation coefficients (Tables 2,3) and coefficients of determination (Figures $1 \mathrm{~J}$ and $4 \mathrm{E}$ ) to climbing ability. Training also increased these variables by $44 \%$ and $49 \%$, respectively, but in this case, climbing ability did not improve (figure 6C-D).

(1)

(1)


This study represents an unprecedented comprehensive effort to identify the physical and physiologic factors that determine performance and enable gains in climbing. We assessed a wide grade-range of climbers from novice lower-grade to dedicated experts in both sexes. This allowed us to characterize the different climbers and identify the main barriers to progression that must be overcome in order to perform at a higher level.

Firstly, in males, $50 \%$ of the assessed parameters correlated with climbing ability. These included, in rank order, shoulder endurance, power and power-endurance, hand and finger strength, hip flexibility or range of motion, lower- and upper-arm strength, core-body endurance, aerobic capacity, hamstrings/lower-limb and lower-back flexibility, and open-hand finger strength. In females, fewer parameters (20\%) correlated with climbing ability. These included, in rank order, shoulder endurance and power, lower-arm strength, balance, aerobic capacity, and arm span ("ape index"), whereas in contrast to males, power-endurance, finger strength, upper-arm strength and flexibility were less important.

Secondly, PCA and multiple regression identified the main determinants of climbing ability. These were shoulder power and endurance, explaining $77 \%$ and $62 \%$ of the variation in climbing ability in males and females, respectively. On further examination, maximum pull-ups, arm cranks, and bent-arm hangs accounted for $59 \%, 14 \%$ and $4 \%$, respectively, of the variation in males, while bent-arm hangs and pull-ups accounted for 54\% and $8 \%$ in females, whereas finger and arm strength also tended to contribute to climbing ability in males. The fact that multiple regression returned only a few significant parameters was expected and may be explained by extensive internal correlations between different variables.

The emphasis on physical capacity of the shoulders as an important determinant for climbing ability, and the further focus on arm, hand and finger strength is in line with previous studies, ${ }^{11,12,19,20}$ but specification of relative contributions is novel. Similarly, flexibility and aerobic capacity contributing to climbing ability has previously received less support; ${ }^{2,4,11,20,21}$ however, a recent report indicated that climbing taxes aerobic capacity sufficient to stress $\mathrm{VO}_{2 \mathrm{max}}{ }^{27}$ and as such this favours a high aerobic capacity in climbers.

It remains unknown why female climbers relied on fewer parameters and were less determined by those, but a possibility is they relied more on factors not assessed here, e.g. technique.

Nonetheless, our approach and analysis represents a step forward for identifying key performance indicators for climbing and thereby informing specific cohorts on training strategies, compared to previous studies where climbing ability has been modelled with less resolution, precision and specificity. ${ }^{5,28}$

The identified determinants and most of the parameters that correlated with climbing ability are trainable and should receive attention in training programs. In a small-scale proof-of-principle training study, we demonstrated that improving the main determinants also improved climbing ability by 2-3 grades. This training was performed in conjunction with other climbing training, which we did not interfere with. Hence, climbing training could explain the observed effect, but this is unlikely since the climbers had a history of $>5$ years of climbing training without experiencing similar gains in performance. In contrast, when variables without significant correlation or regression to climbing ability (balance and leg-raise hang) improved by similar magnitudes, climbing ability did not improve. This strengthens the role of the main determinants identified here as key performance indicators, though the full effect of training should be studied in more depth. Our results also suggest, with the possible exception for hand and arm reach, that non-trainable anthropometric and body dimension factors matter less for climbing ability, as previously observed. ${ }^{5,11,17,20}$ Thus, gains in climbing may be less restricted by specific body- or somatotypes; however, we caution that the subjects in this study were homogeneous with respect to body mass and dimensions, and neither was the study designed to investigate this aspect. 
Notwithstanding the above, a successful training program should be balanced and seek to improve a wide spectrum of physical and physiologic capacities, especially if pursuing climbing of various styles. We must however point out that the recruitment of climbers to our study via an indoor climbing facility with mainly short and steep (overhanging) routes may have favoured shoulder endurance, power, and power-endurance as the main determinants of climbing ability. ${ }^{3}$ Other subdisciplines or climbing styles not investigated here may differ, and we did not assess technique, economy, recovery or resistance to fatigue, which may all contribute toward climbing ability. ${ }^{12,15,16,23,29,30}$ Finally, although we balanced numbers and sought to recruit at least 3 subjects in each grade in order to achieve the necessary statistical power, reduced availability of elite climbers restricted numbers in the highest grades. This may have limited the analysis.

\section{Practical Applications}

Several important practical applications may be deduced from this study: 1) improvements to shoulder power and endurance and to a lesser extent finger and arm strength will improve climbing performance; 2) these and further determinants of climbing are trainable; and 3) body weight and dimensions do not stop progress in climbing. These applications relate to all climbers regardless of ability or sex, but may especially pertain to those that climb relatively short, but steep routes.

\section{Conclusions}

Peak performance in climbing is achieved at least partly as a result of well-developed physical and physiologic characteristics, and high gains are accomplished by focused and dedicated training that improves those characteristics. Here, we have identified the characteristics that need be overcome for continued progression and success. They include shoulder endurance, power and power-endurance, hand and finger strength, lower- and upper-arm strength, flexibility, core-body endurance, balance, and aerobic capacity, with shoulder power and endurance emerging as the main determinants. These key performance indicators should be included in training for climbing and if improved, will lead to improved performance.

\section{ACKNOWLEDGEMENTS}

The authors thank the climbers and Glasgow Climbing Centre for their contributions.

(1)

(1)

(1)

(1)


416

417

418

419

420

421

422

423

424

425

426

427

428

429

430

431

432

433

434

435

436

437

438

439

440

441

442

443

444

445

446

447

448

449

450

451

452

453

454

455

456

457

458

459

460

461

462

463

464

465

466

1. Mermier CM, Robergs RA, McMinn SM, Heyward VH. Energy expenditure and physiological responses during indoor rock climbing. Br J Sports Med. 1997;31:224-228.

2. Booth J, Marino F, Hill C, Gwinn T. Energy cost of sport rock climbing in elite performers. Br J Sports Med. 1999;33:14-18.

3. De Geus B, Villanueva O’Driscoll S, Meeusen R. Influence of climbing style on physiological responses during indoor rock climbing on routes with the same difficulty. Eur $J$ Appl Physiol. 2006;98:489-496.

4. Billat V, Palleja P, Charlaix T, Rizzardo P, Janel N. Energy specificity of rock climbing and aerobic capacity in competitive sport rock climbers. J Sports Med Phys Fitness. 1995;35:2024.

5. Laffaye G, Levernier G, Collin JM. Determinant factors in climbing ability: influence of strength, anthropometry, and neuromuscular fatigue. Scand J Med Sci Sports. 2016;26:11511159 .

6. Laffaye G, Collin JM, Levernier G, Padulo J. Upper-limb power test in rock-climbing. Int J Sports Med. 2014;35:670-675.

7. Watts PB, Newbury V, Sulentic J. Acute changes in handgrip strength, endurance, and blood lactate with sustained sport rock climbing. J Sports Med Phys Fitness 1996;36:255-260.

8. Watts PB. Physiology of difficult rock climbing. Eur J Appl Physiol. 2004;91:361-372.

9. Draper N, Dickson T, Blackwell G, Priestley S, Fryer S, Marshall H, Shearman J, Hamlin M, Winter D, Ellis G. Sport-specific power assessment for rock climbing. J Sports Med Phys Fitness. 2011;51:417-425.

10. Balas J, Panackova M, Strejcova B, Martin AJ, Cochrane DJ, Kalab M, Kodejska J, Draper N. The relationship between climbing ability and physiological responses to rock climbing. Sci World J. 2014;27:678387.

11. Grant S, Hasler T, Davies C, Aitchison TC, Wilson J, Whittaker A. A comparison of the anthropometric, strength, endurance and flexibility characteristics of female elite and recreational climbers and non-climbers. J Sports Sci. 2001;19:499-505.

12. MacLeod D, Sutherland DL, Buntin L, Whitaker A, Aitchison T, Watt I, Bradley J, Grant S. Physiological determinants of climbing-specific finger endurance and sport rock climbing performance. J Sports Sci. 2007;25:1433-1443.

13. Philippe M, Wegst D, Muller T, Raschner C, Burtscher M. Climbing-specific finger flexor performance and forearm muscle oxygenation in elite male and female sport climbers. Eur $J$ Appl Physiol. 2012;112:2839-2847.

14. Espana-Romero V, Ortega Porcel FB, Artero EG, Jimenez-Pavon D, Gutierrez Sainz A, Castillo Garzon MJ, Ruiz JR. Climbing time to exhaustion is a determinant of climbing performance in high-level sport climbers. Eur J Appl Physiol. 2009;107:517-525.

15. Fryer S, Stoner L, Scarrott C, Lucero A, Witter T, Love R, Dickson T, Draper N. Forearm oxygenation and blood flow kinetics during a sustained contraction in multiple ability groups of rock climbers. J Sports Sci. 2015;33:518-526.

16. Fryer S, Stoner L, Stone K, Giles D, Sveen J, Garrido I, Espana-Romero V. Forearm muscle oxidative capacity index predicts sport rock-climbing performance. Eur J Appl Physiol. 2016;116:1479-1484.

17. Watts PB, Martin DT, Durtschi S. Anthropometric profiles of elite male and female competitive sport rock climbers. J Sports Sci. 1993;11:113-117.

18. Watts PB, Joubert LM, Lish AK, Mash JD, Wilkins B. Anthropometry of young competitive sport rock climbers. Br J Sports Med. 2003;37:420-424.

19. Ferguson RA, Brown MD. Arterial blood pressure and forearm vascular conductance responses to sustained and rhythmic isometric exercise and arterial occlusion in trained rock climbers and untrained sedentary subjects. Eur J Appl Physiol. 1997;76:174-180.

20. Mermier CM, Janot JM, Parker DL, Swan JG. Physiological and anthropometric determinants of sport climbing performance. Br J Sports Med. 2000;34:359-365. 
21. Sheel AW, Seddon N, Knight A, McKenzie DC, Warburton DER. Physiological responses to indoor rock-climbing and their relationship to maximal cycle ergometry. Med Sci Sports Exerc. 2003;35:1225-1231.

22. Draper N, Dickson T, Blackwell G, Fryer S, Priestley S, Winter D, Ellis G. Self-reported ability assessment in rock climbing. J Sports Sci. 2011;29:851-858.

23. Draper N, Jones GA, Fryer S, Hodgson C, Blackwell G. Effect of an on-sight lead on the physiological and psychological responses to rock climbing. J Sports Sci Med. 2008;7:492498.

24. Martens R, Vealey RS, Burton D, Bump L, Smith DE. Development and validation of the competitive state anxiety inventory-2. In: Martens R, Vealey RS, Burton D, eds. Competitive anxiety in sport. Champaign, IL: Human Kinetics; 1990:117-178.

25. Torpel A, Becker T, Thiers A, Hamacher D, Schega L. Intersession reliability of isokinetic strength testing in knee and elbow extension and flexion using the BTE PrimusRS. $J$ Sport Rehabil. 2017;26:doi:10.1123/jsr.2016-0209.

26. Devlin J, Paton B, Poole L, Sun W, Ferguson C, Wilson J, Kemi OJ. Blood lactate clearance after maximal exercise depends on active recovery intensity. J Sports Med Phys Fitness. 2014:54:271-278.

27. Limonta E, Brighenti A, Rampichini S, Ce E, Schena F, Esposito F. Cardiovascular and metabolic responses during indoor climbing and laboratory cycling exercise in advanced and élite climbers. Eur J Appl Physiol. 2018;118:371-379.

28. Magiera A, Roczniok R, Maszczyk A, Czuba M, Kantyka J, Kurek P. The structure of performance of a sport rock climber. J Hum Kinet. 2013;36:107-117.

29. Bertuzzi RC, Franchini E, Kokubun E, Kiss MA. Energy system contributions in indoor rock climbing. Eur J Appl Physiol. 2007;101:293-300.

30. Sibella F, Frosio I, Schena F, Borghese NA. 3D analysis of the body center of mass in rock climbing. Hum Mov Sci. 2007;26:841-852.

\section{4}

(1)

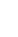


Figure 1. Relationships and coefficients of determination $\left(\mathrm{r}^{2}\right)$ between climbing ability and maximum achievable pull-ups (A and $B$ ), bent-arm hang-time ( $C$ and $D)$, average 20 seconds arm crank power ( $E$ and $F$ ), maximum arm crank power ( $G$ and $H)$, and leg-raise hang-time (I and J), in male (left) and female (right) climbers, respectively. Insets show relationships after normalizing for body weight. Individual data with linear regression.

Figure 2. Relationships and coefficients of determination $\left(r^{2}\right)$ between climbing ability and maximum arm bicep strength (A and $B$ ), arm grip strength (C and $D)$, finger pincer strength ( $\mathrm{E}$ and $\mathrm{F}$ ), and open-hand finger strength ( $\mathrm{G}$ and $\mathrm{H}$ ), in male (left) and female (right) climbers, respectively. Insets show relationships after normalizing for body weight. Individual data with linear regression.

Figure 3. Relationships and coefficients of determination $\left(\mathrm{r}^{2}\right)$ between climbing ability and aerobic capacity, measured as upper-body peak oxygen uptake $\left(\mathrm{VO}_{2 \text { peak }}\right.$; $\mathrm{A}$ and $\left.\mathrm{B}\right)$ and wholebody maximal oxygen uptake $\left(\mathrm{VO}_{2 \max } ; \mathrm{C}\right.$ and $\left.\mathrm{D}\right)$, in male (left) and female (right) climbers, respectively. Individual data with linear regression.

Figure 4. Relationships and coefficients of determination $\left(\mathrm{r}^{2}\right)$ between climbing ability and flexibility, measured as leg-span for hip flexibility (A and B) and sit-and-reach test for hamstring and lower-back flexibility (C and D), and balance ( $\mathrm{E}$ and F), in male (left) and female (right) climbers, respectively. Insets show relationships after normalizing for body height. Individual data with linear regression.

Figure 5. Relationships and coefficients of determination $\left(\mathrm{r}^{2}\right)$ between climbing ability and body dimensions, measured as body weight ( $\mathrm{A}$ and $\mathrm{B}$ ) and body height ( $\mathrm{C}$ and $\mathrm{D})$ in male (left) and female (right) climbers, respectively, as well as arm span in male climbers (E) and "ape index " (arm span/body height) in female climbers (F). Individual data with linear regression.

Figure 6. Principal component analysis (PCA) and multiple univariate stepwise linear regression identified the main explanatory variables that determined climbing ability. Males (A): maximum pull-ups, average arm crank power, and bent-arm hang: unstandardized coefficients $b 0.48 \pm$ standard error (SE) 0.06, 0.03 \pm SE 0.01, and 0.06 \pm SE 0.02, respectively, adjusted $R^{2} 0.77 ; 0.59,0.14$, and 0.04 , respectively, and residual SD 1.73. Females (B): bentarm hang and maximum pull-ups: unstandardized coefficients $b 0.14 \pm$ SE $0.02,0.22 \pm$ SE 0.08, respectively, adjusted $R^{2} 0.62 ; 0.54$, and 0.08 , respectively, and residual SD 1.76 . When males $(C)$ trained maximum pull-ups $(n=3)$ or balance $(n=3)$, and females $(D)$ trained bentarm hangs $(n=3)$ or leg-raise hangs $(n=3)$ for 8 weeks, the independent variables increased (left $\mathrm{Y}$-axis), but climbing grade only increased when main determinants maximum pull-ups (males) or bent-arm hangs (females) increased (right Y-axis), and not when balance or legraise hangs increased. 